\title{
Initial Results from the CryoARM300 and DE-64 Counting
}

Benjamin Bammes ${ }^{1 *}$, Michael Spilman ${ }^{1}$, James Streetley $^{2}$ and David Bhella ${ }^{2}$

${ }^{1 .}$ Direct Electron, San Diego, CA, USA.

2. Scottish Centre for Macromolecular Imaging, University of Glasgow, Glasgow, UK.

* Corresponding author: bbammes@directelectron.com

Over the past five years, electron cryo-microscopy (cryo-EM) has become the most popular and effective technique in structural biology, due to the hardware and software innovations that delivered cryo-EM's "resolution revolution" [1]. However, there are still significant opportunities for further improvement, especially with respect to maximizing data quality and throughput to satisfy the high demand for cryoEM resources and alleviate the high cost of purchasing, maintaining, and operating cryo-EM equipment.

We have evaluated a new CryoARM300 transmission electron microscope (TEM) with a DE-64 Camera. This combination promises two primary advantages for cryo-EM data quality: First, the cold fieldemission gun (FEG) should improve the coherence of the electrons in the column and improve the resolution of the microscope. Second, counting mode on the DE-64 Camera should have high modulation transfer function (MTF) and detective quantum efficiency (DQE) due to its unique CMOS pixel design.

Monte Carlo simulations [2] of $300 \mathrm{kV}$ primary electrons incident on a $30 \mu \mathrm{m}$ thick silicon layer (representing the total thickness of a CMOS direct detection active pixel sensor) show that approximately $75 \%$ of electrons scatter less than $6.5 \mu \mathrm{m}$ from the point of incidence on the detector as they traverse the silicon layer. However, because the scattering of each electron is stochastic, primary electrons that scatter across multiple pixels on the sensor cannot be unambiguously localized to a single point of incidence on the sensor. Therefore, optimal resolution on the sensor is achieved through the use of large pixels, so that primary electrons deposit signal on one and only one pixel. Operating the DE-64 Camera with $2 \times$ hardware binning effectively delivers a $4 \mathrm{k} \times 4 \mathrm{k}$ direct detection sensor with $13 \mu \mathrm{m}$ pixels, which is large enough that the majority of $300 \mathrm{kV}$ primary electron deposit signal in one and only one pixel. Measurements of the modulation transfer function (MTF) [3] show electron counting in this mode provides near optimal resolution on the sensor (Fig. 1, left).

Because the electron exposure is limited in cryo-EM due to radiation damage [4], detecting the precise location of primary electrons is not helpful if the detector either misses many primary electrons (false negatives) or has high noise causing false positives. In a conventional CMOS image sensor, each pixel essentially has three sequential operations: reset, acquire, and readout. Noise in the reset operation is added to signal readout from each pixel, resulting in noisy images and false positives during electron counting. This problem can be resolved by implementing correlated double sampling (CDS), where the pixel value is immediately sampled after each reset operation prior to the acquisition of real signal in the pixel. This baseline signal is then subtracted from the final readout signal of each pixel, effectively removing reset noise. Operating the DE-64 with on-chip CDS results in very low RMS background noise so that the threshold for electron counting can be effectively set to minimize both false positives and false negatives. Based on the curves fit to histograms of the pixel values from unilluminated pixels and pixel values corresponding to primary electron events, the threshold on the DE-64 Camera effectively discards $99.9 \%$ of background noise while detecting $\sim 99.8 \%$ of electron events incident on the sensor (Fig. 1, right). 
We have demonstrated the use of the CryoARM300 with cold-FEG and the DE-64 Camera operating in electron counting mode by carrying out automated single-particle cryo-EM data acquisition of apoferritin. Data was acquired with JADAS automation software [5]. Reconstruction of this initial data set with Relion [6] yielded a $3.6 \AA$ resolution structure (Fig. 2), demonstrating an effective workflow for cryo-EM data acquisition with this equipment. With this workflow now established, we can now test the limits of data quality and throughput on the CryoARM300 and DE-64 Camera [7].

References:

[1] W Kühlbrandt, Science 343 (2014), p. 1443.

[2] J Baró et al., Nucl. Instrum. Meth. B 100 (1995), p. 31.

[3] RS Ruskin, Z Yu and N Grigorieff, J. Struct. Biol. 184 (2013), p. 385.

[4] BE Bammes et al., J. Struct. Biol. 169 (2010), p. 331.

[5] J Zhang et al., J. Struct. Biol. 165 (2009), p. 1.

[6] SHW Scheres, J. Struct. Biol. 180 (2012), p. 519.

[7] The authors acknowledge Andy Yarwood at JEOL UK for assistance setting up JADAS.
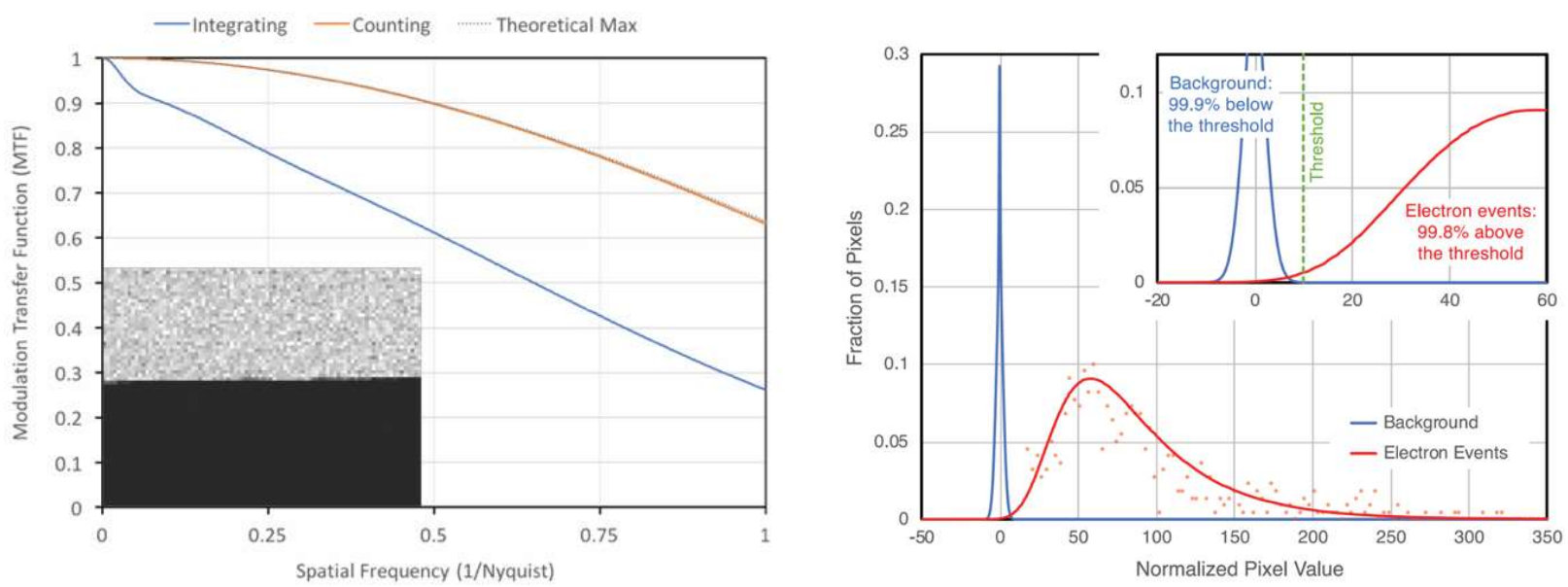

Figure 1. (Left) The MTF of the DE-64 in integrating mode (unbinned) and counting mode (2x-hardwarebinned). The inset shows a portion of an image of the edge of a beamstop in counting mode. (Right) Histograms of the background pixel counts (blue) and foreground pixel counts (red) on the DE-64 with $2 \mathrm{x}$-hardware-binning. The inset shows the negligible overlap between the two histograms and the threshold value (green) used for distinguishing electron events from background in counting mode.
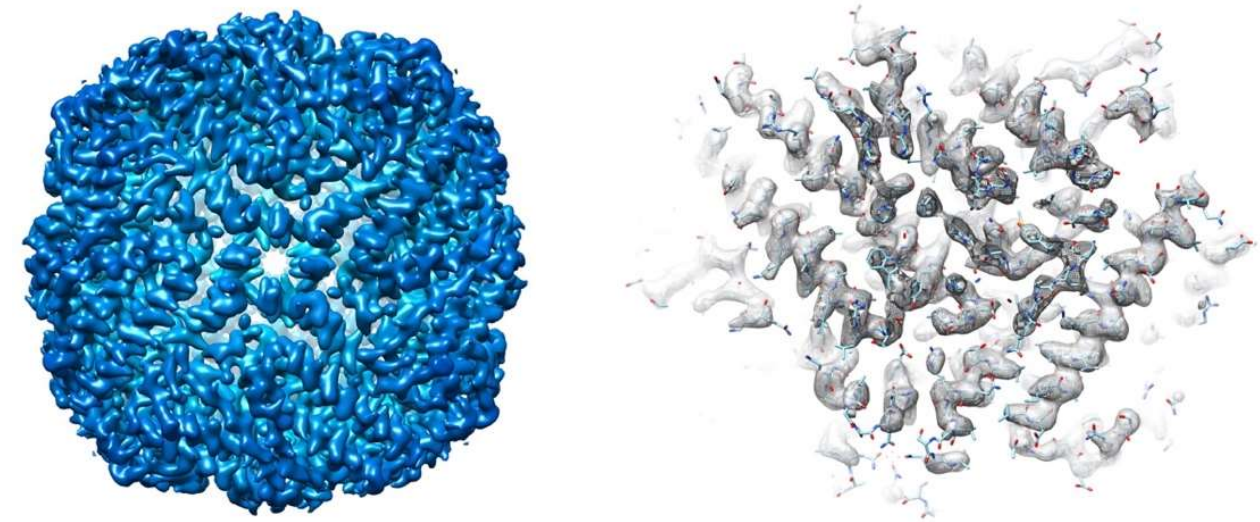

Figure 2. Views of the initial 3D reconstruction of apoferritin. 